\title{
Genetic mapping and developmental timing of transmission ratio distortion in a mouse interspecific backcross
}

\author{
Chevonne D Eversley ${ }^{1}$, Tavia Clark', Yuying Xie ${ }^{1}$, Jill Steigerwalt ${ }^{1,2}$, Timothy A Bell ${ }^{1}$, \\ Fernando PM de Villena ${ }^{1}$, David W Threadgill ${ }^{1,2^{*}}$
}

\begin{abstract}
Background: Transmission ratio distortion (TRD), defined as statistically significant deviation from expected 1:1 Mendelian ratios of allele inheritance, results in a reduction of the expected progeny of a given genotype. Since TRD is a common occurrence within interspecific crosses, a mouse interspecific backcross was used to genetically map regions showing TRD, and a developmental analysis was performed to identify the timing of allele loss.

Results: Three independent events of statistically significant deviation from the expected 50:50 Mendelian inheritance ratios were observed in an interspecific backcross between the Mus musculus A/J and the Mus spretus SPRET/EiJ inbred strains. At weaning M. musculus alleles are preferentially inherited on Chromosome (Chr) 7, while M. spretus alleles are preferentially inherited on Chrs 10 and 11. Furthermore, alleles on Chr 3 modify the TRD on Chr 11. All TRD loci detected at weaning were present in Mendelian ratios at mid-gestation and at birth.

Conclusions: Given that Mendelian ratios of inheritance are observed for Chr 7, 10 and 11 during development and at birth, the underlying causes for the interspecific TRD events are the differential post-natal survival of pups with specific genotypes. These results are consistent with the TRD mechanism being deviation from Mendelian inheritance rather than meiotic drive or segregation distortion.
\end{abstract}

\section{Background}

Commonly used inbred mouse strains, which trace their genetic ancestry primarily to the Mus musculus domesticus subspecies [1], have extensive interspecific polymorphic differences when compared to Mus spretus. Because of the large number of polymorphisms that are distributed across the genome, interspecific crosses are frequently used to map genes responsible for variation in a variety of phenotypic traits [2]. In crosses between $M$. musculus and M. spretus only interspecific backcrosses using hybrid females are possible since hybrid males are sterile. However, interspecific backcrosses often result in skewed distributions in the inheritance of polymorphic alleles from the hybrid females, a phenomenon called transmission ratio distortion (TRD) [3-8].

\footnotetext{
* Correspondence: threadgill@ncsu.edu

'Department of Genetics, Curriculum in Genetics and Molecular Biology, and Carolina Center for Genome Sciences, University of North Carolina, Chapel Hill, NC 27599, USA

Full list of author information is available at the end of the article
}

Transmission ratio distortion is defined as statistically significant deviation from the expected 1:1 Mendelian ratios of allele inheritance, resulting in a reduction of the expected progeny of a given genotype.

Transmission ratio distortion involving $M$. spretus crosses was first identified during linkage testing on Chromosomes (Chrs) 2, 4 and 10 [8-11]. Subsequent efforts attempted to map the causative loci influencing TRD in four backcrosses involving M. spretus [6]. Transmission ratio distortion has also been observed in wild M. musculus populations involving Chr 1 and in commonly derived inbred strains on Chr 11 [12-15].

Among the causes of TRD are meiotic drive, segregation distortion (SD), and deviation from Mendelian inheritance (DMI) [6]. The defining characteristic of meiotic drive is that TRD occurs during female meiosis [16]. Consequently, the resulting gametes are not lost and fertility is unaffected, but the inheritance of adjacent neutral polymorphisms is affected $[17,18]$. Meitoic drive is one of the more common examples in which a "selfish 
gene" drives the preferential selection and fertilization of an oocyte [6,17]. An example of meiotic drive at the second meiotic division can be seen in the DDK syndrome at the Om locus on mouse Chr 11 [19,20].

Segregation distortion is due to a chromosomal transmission imbalance that typically occurs after meiosis but prior to fertilization. This mechanism is responsible for the $S D$ system in Droshophila melanogaster and the mouse $t$-haplotype [21-25]. Finally, DMI occurs as a result of post-fertilization lethality of embryos or neonates with a particular genotype. Therefore, DMI can be used to map loci at which specific alleles have detrimental effect on survival. This is particularly interesting in crosses between closely related species because DMI may provide an important tool to study the genetics of speciation.

In this study we report three independent occurrences of TRD caused by post-meiotic lethality in a single interspecific backcross population between A/J (M. musculus) and SPRET/EiJ (M. spretus) mouse inbred strains. Preferential transmission of $M$. musculus alleles is observed on Chr 7 and of M. spretus alleles on Chrs 10 and 11. In addition, the Chr 11 TRD is modified by a locus on Chr 3. All three loci showing TRD are consistent with a DMI cause since allele-specific losses are not observed until after birth.

\section{Results}

The number of progeny inheriting $\mathrm{S}$ or A alleles from an ASF1 female backcrossed to an A male was used to measure transmission frequencies across the mouse genome and to detect TRD. Three genomic intervals were detected that showed non-Mendelian inheritance (Table 1). Transmission ratio distortion favoring A alleles was observed on Chr $7\left(\chi^{2}=7.87\right.$; $\left.\mathrm{p}=0.005\right)$, while elevated frequencies of $\mathrm{S}$ alleles were observed on Chr $10\left(\chi^{2}=30.68 ; \mathrm{p}=3.0 \times\right.$ $\left.10^{-8}\right)$ and Chr $11\left(\chi^{2}=19.93 ; \mathrm{p}=8.0 \times 10^{-6}\right)$. There was no difference in TRD presence and level between female and male progeny (data not shown).

An approach developed to map TRD to a single locus or multiple linked loci was used to identify the location of the distorted loci with the minimum goodness-of-fit (GF) for each TRD region [6,19]. The distribution of allele frequencies along Chr 7 can be explained by TRD at a single locus located within a $6 \mathrm{cM}$ interval centered at $27.8 \mathrm{cM}$ (Figure 1A). The best GF location was determined by incrementally shifting $0.2 \mathrm{cM}$ away from rs8260829 at $28 \mathrm{cM}$ (GF $=10.003,31$ d.f., not significant $\mathrm{p}=0.99)$.

Among 19 SNPs on Chr 10, a single peak was evident at $r s 4228380$ (Figure 1B). The model posits that a single distorted locus is located at $48.5 \mathrm{cM}$ with an expected distortion of $71 \%$. This is in good agreement with the predictions of the GF model ( $G F=12.08,19$ d.f., not significant $\mathrm{p}=0.88)$. Incremental adjustments of the location and TRD had no affect on the minimum GF.

Chromosome 11 shows broad distortion spanning 16 SNPs incrementally spaced with a maximum peak of $67 \%$ TRD (Figure 1C). After adjusting the location of GF and TRD, the best GF was found to be near rs13481119 at $45 \mathrm{cM}$ distal to the centromere (GF = 3.631, 16 d.f., not significant $\mathrm{p}=0.99$ ).

In addition to reduced fitness of gametes inheriting the A allele on Chr 11, transmission of alleles on Chr 11 was strongly modified by co-segregating alleles on Chr 3 (Table 2). Gametes inheriting A alleles on both Chr 3 and 11 showed significantly reduced fitness. The strongest interaction occurred between a locus at approximately $4.6 \mathrm{cM}$ distal to the centromere on Chr 3 and $46.0 \mathrm{cM}$ distal to the centromere on Chr $11\left(\chi^{2}=\right.$ $11.89, \mathrm{p}=0.0005)$. No interactions with other regions of the genome were detected for TRD on Chrs 7 or 10 .

Post-weaning mortality was minimal and could not account for the deficit of specific alleles in the TRD intervals, indicating that allele loss leading to TRD occurs before weaning. To determine the timing of allele loss, (ASF1)A neonates and embryos were produced and TRD intervals genotyped using informative microsatellite markers. Mendelian ratios were observed in embryos and in neonates for each of the three TRD intervals (Table 3). These data show that the TRD is not a result of a meiotic selection in the ASF1 heterozygous dam or a preferential survival of embryos. In addition, there was no correlation between placenta or birth weight and genotype (data not shown). Although genotyping was not performed on adult mice, these experiments were conducted using inbred mouse strains at the same facility under the same conditions therefore we expect DMI to be present and reproducible. Consequently, the TRD for loci on Chrs 7, 10 and 11 in the interspecific backcross is caused by DMI that occurs post-natally between birth and weaning.

Table 1 SNP markers displaying TRD

\begin{tabular}{|c|c|c|c|c|c|c|c|}
\hline \multirow[b]{2}{*}{ Chromosome } & \multirow[b]{2}{*}{ Marker } & \multicolumn{2}{|c|}{ Observed } & \multirow[b]{2}{*}{ Expected } & \multicolumn{2}{|l|}{ A allele } & \multirow[b]{2}{*}{ p-value } \\
\hline & & AA & AS & & Transmission & $\chi^{2}$ & \\
\hline Chr 7 & rs8260829 & 139 & 96 & 117.5 & $59.1 \%$ & 7.87 & 0.005 \\
\hline Chr 10 & rs4228380 & 73 & 157 & 115 & $31.7 \%$ & 30.68 & 3.0E-08 \\
\hline Chr 11 & rs3707772 & 82 & 150 & 116 & $35.3 \%$ & 19.93 & 8.0E-06 \\
\hline
\end{tabular}




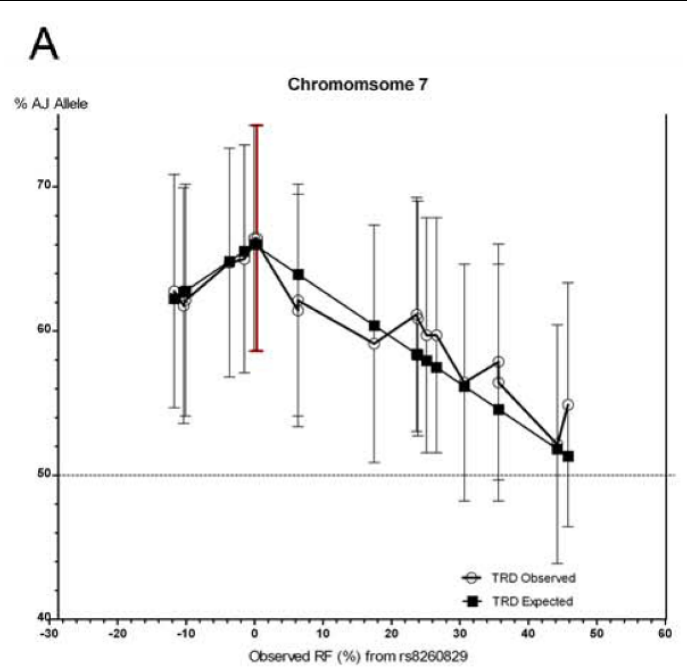

B

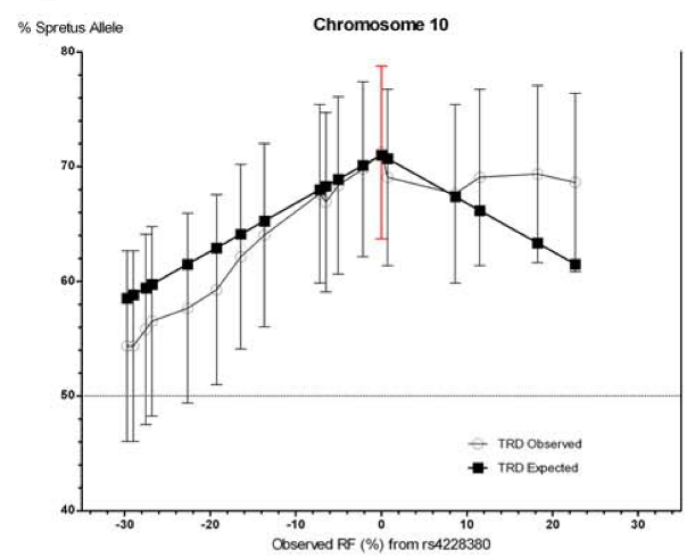

C

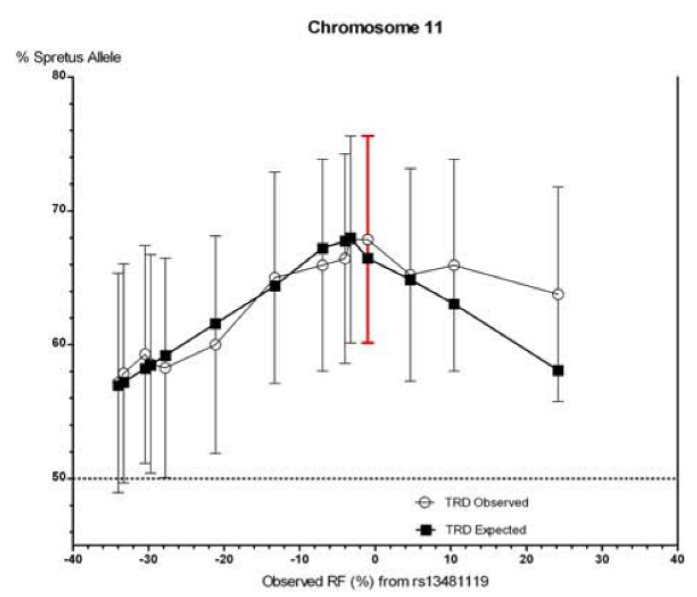

Figure 1 Genetic maps of TRD. (A) Percent transmission of A alleles when fit to a single-locus model at rs8260829 on Chr 7. (B) Percent transmission of S alleles when fit to a single-locus model at rs4228380 on Chr 10. (C) Percent transmission of S alleles when fit to a single-locus model at rs13481119 on Chr 11. Open circles represent the distortion observed. Darkened squares represent the level of distortion expected with a single distorting locus positioned at the maximally distorted marker on each chromosome. Red lines indicate maximal likely location of the TRD causing alleles. The observed recombination distances are indicated on the $\mathrm{x}$-axis and only markers showing distortion are represented. 
Table 2 SNP markers showing an interaction influencing TRD

\begin{tabular}{cccccc}
\hline & & \multicolumn{2}{c}{ Chr 3 rs3694133 } & $\chi^{2}$ & p-value \\
& AA & AS & & \\
\hline Chr 11 & AA & 15 & 30 & 11.89 & 0.0005 \\
rs13481119 & AS & 60 & 33 & & \\
\hline
\end{tabular}

\section{Discussion}

Three independent loci causing strong transmission ratio distortion favoring A alleles on Chr 7 and $\mathrm{S}$ alleles on Chrs 10 and 11 were identified. This is the third report of TRD involving Chr 11, but the first to document an interaction with Chr $3[12,14]$. The distorting losses all occur after birth but prior to weaning and are likely the result of allelic incompatibilities influencing pup survival.

The region of maximum distortion on Chr 7 has been reported to harbor many imprinted genes influencing fetal and placental growth, postnatal growth, lethality and viability [26]. Most notably, this region contains orthologs of the imprinted gene cluster involved in the neurological disorders Prader-Willi Syndrome and Angelman Syndrome; Prader-Willi Syndrome is caused by the lack of a functional paternal copy (maternally imprinted), while Angelman Syndrome is caused by a lack of maternal copy [27]. Investigation into the genes underlying Prader-Willi Syndrome reveals that Necdin mutants display respiratory instability and die within the first week after birth [28]. Forty-five percent of mice with maternally transmitted Angelman Syndrome die seven days after birth or display a reduction in postnatal growth and viability $[29,30]$.

It is more plausible that the distortion evident in this study was due to maternally inherited chromosomes

Table 3 Timing of the TRD effect

\begin{tabular}{lccccc}
\hline Marker & \multicolumn{2}{c}{ Observed } & Expected & $\boldsymbol{\chi}^{\mathbf{2}}$ & p-value \\
& AS & AA & & & \\
\hline Pre-natal & & & & & \\
D7Mit309 & 28 & 31 & 29.5 & 0.15 & 0.70 \\
D10Mit108 & 30 & 30 & 30 & 0.00 & 1.00 \\
D10Mit145 & 28 & 33 & 30.5 & 0.41 & 0.52 \\
D11Mit152 & 27 & 31 & 29 & 0.28 & 0.60 \\
D11Mit225 & 31 & 28 & 29.5 & 0.15 & 0.70 \\
D11Mit338 & 35 & 25 & 30 & 1.67 & 0.20 \\
Post-natal & & & & & \\
D7Mit309 & 42 & 44 & 43 & 0.05 & 0.83 \\
D10Mit108 & 47 & 37 & 42 & 1.19 & 0.28 \\
D10Mit145 & 46 & 41 & 43.5 & 0.29 & 0.59 \\
D11Mit152 & 38 & 48 & 43.5 & 1.39 & 0.24 \\
D11Mit225 & 42 & 46 & 44 & 0.18 & 0.67 \\
D11Mit338 & 40 & 43 & 43 & 0.42 & 0.52 \\
\hline
\end{tabular}

with a paternal imprint. Angelman Syndrome, rather than Prader-Willi Syndrome, is caused by a loss of maternal genetic contribution. Although, Prader-Willi Syndrome causes postnatal lethality, the phenotype of Angelman Syndrome is not $100 \%$ penetrant and is consistent with the abundance of homozygous animals in the interspecific backcross. The TRD on Chr 7 could be due to variants within the orthologous Angelman Syndrome imprinting center or strain-specific methylation patterns causing silencing of maternally transmitted S allele(s). Meiotic transmission of the A allele by the heterozygous mother results in a viable homozygous mouse and is unlikely to be influenced by methylated silencing. Imprinting defects have been reported in interspecific crosses [31-33], and disregulation of imprinting is thought to be common cause of hybrid disgenesis [34].

The TRD on Chr 10 has been previously reported using C57BL/6J and SPRET/EiJ backcrosses [7,10]; the genetic similarity between $\mathrm{A} / \mathrm{J}$ and $\mathrm{C} 57 \mathrm{BL} / 6 \mathrm{~J}$ suggests that the distorted locus is conserved in these strains [35]. In previous reports the Chr 10 TRD was reported to extend from $M y b$ to Ifg1 with an apex at Col6a1/ Col6a, near D10Mit242 (41.2 cM) [10]. A similar TRD spanning more than $15 \mathrm{cM}$ and with maximum peak at D10Bir9 (30 cM) was also reported for an interspecific backcross [7]. Reciprocal crosses confirmed a preferential selection of heterozygous alleles over homozygous C57BL/6J alleles similar to the data presented here.

Unlike Chr 7, the TRD interval on Chr 10 is not known to contain imprinted genes. It is possible that the TRD on Chr 10 may be influenced by global strainspecific methylation differences resulting in an incompatibility that is not completely penetrant.

Although epistatic interactions influencing TRD have been reported $[6,36,37]$, the current data reports an independent modifier that is not located in a region of statistically significant distortion yet has a striking effect on the Chr 11 TRD. Transmission ratio distortion on Chr 11 is increased among backcross progeny homozygous for A/J genotypes on Chr 3 suggesting a maladaptive incompatibility between $C h r 3^{A A}$ and $C h r 11^{A A}$. There is a possibility for a third undetected modifier influencing the TRD of Chr 11 since the least represented progeny are $C h r 3^{A A}$, Chr $11^{A A}$.

Although there are now two reports of TRD occurring in a similar region on Chr 11, the data suggests different mechanism are responsible. Unlike the previous report, which reported a sex skewed TRD without a Chr 3 interaction in a cross between two M. musculus-derived strains [12], the TRD of Chr 11 reported here does not differentially affect the sexes. Additionally, it is unlikely to be due to the early embryonic lethal DDK syndrome since the timing of TRD is between birth and weaning. 
Further phenotypic characterization of developing pups will be required to identify the physiological cause of DMI on Chrs 7, 10 and 11 in mouse interspecific backcrosses.

\section{Conclusion}

In summary, Mendelian ratios of inheritance occur for Chr 7, 10 and 11 during development and at birth, but not at weaning. Additionally, Chr 3 genotypes influence TRD of Chr 11. The data indicate a differential postnatal survival of pups with specific genotypes. These results are consistent with the TRD mechanism being deviation from Mendelian inheritance rather than meiotic drive or segregation distortion.

\section{Methods}

\section{Genetic crosses}

All mice were obtained from The Jackson Laboratory (Bar Harbor, ME). Female A/J (A) mice (M. musculus) were crossed to male SPRET/EiJ (S) mice (M. spretus) to generate interspecific hybrids. In all crosses dams are listed first and sire second. Female ASF1 hybrids were backcrossed to $\mathrm{A} / \mathrm{J}$ males to generate a segregating population of 235 (ASF1)A backcross mice in the initial experiment. Mice were euthanized at eight months of age and DNA extracted from tail and liver tissue using a Purgene DNA Extraction kit (Promega, Madison, WI).

Additional cohorts of (ASF1)A offspring were generated and euthanized within two days of birth $(n=88)$, or pre-natally between $12.5-19.5$ days post-coitus $(n=$ 60). For pre-natal samples, placenta and embryo weights were recorded and DNA was extracted from tail tissue by phenol-chloroform extraction.

All animal studies were approved by the Institutional Animal Care and Use Committee of University of North Carolina at Chapel Hill.

\section{Genotyping}

DNA samples from (ASF1)A mice were commercially genotyped using 254 informative single nucleotide polymorphisms (SNP) markers (Illumina, San Diego, CA). Additional genotyping was done using a custom Sequenom MassArray SNP Genotyping platform containing 182 SNP markers (Geneseek, Lincoln, NE). Sequenom SNP markers were selected from NCBI Build 37 placed at approximately 10-15 cM intervals (Additional file 1, Table S1).

The microsatellite markers D7Mit309, D10Mit145, D10Mit108, D11Mit338, D11Mit152 and D11Mit225 (Operon Biotechnologies, Huntsville, AL) were used to test for TRD in embryos and neonates. Standard PCR methods were used $[38,39]$. PCR products were fractionated using $2 \%-4 \%$ agarose gels and stained with ethidium bromide for visualization.

\section{Statistical analysis}

Evaluation of loci for TRD was performed on each cross using Chi square analysis with one degree of freedom. A corrected version of Montagutelli's GF test for single or multiple loci showing TRD was used as previously described $[6,19]$. A correlation analysis was performed to detect associations between genotype and placenta or birth weight. Pair-wise analyses were also performed genome-wide with the primary TRD loci to detect modifier loci. Significance thresholds were corrected for multiple testing [40].

\section{Additional material}

Additional file 1: Markers used in genetic mapping. Table listing of the SNP markers used in the genetic analysis.

\section{Acknowledgements}

The authors thank members of our lab for assistance and helpful discussions. CDE was supported by pre-doctoral fellowship CA123636 from the National Institutes of Health. DWT acknowledges support by National Institutes of Health grants CA079869 and CA105417.

\section{Author details}

${ }^{1}$ Department of Genetics, Curriculum in Genetics and Molecular Biology, and Carolina Center for Genome Sciences, University of North Carolina, Chapel Hill, NC 27599, USA. ²Department of Genetics, North Carolina State University, Raleigh, NC 27695, USA.

\section{Authors' contributions}

Conceived and designed the experiments: CDE and DWT. Performed the experiments: CDE, TC, YX, JS and TAB. Analysed the data: CDE, YX, FPMV and DWT. Wrote the paper: CDE, FPMV and DWT. All authors read and approved the final manuscript.

Received: 23 May 2010 Accepted: 3 November 2010 Published: 3 November 2010

\section{References}

1. Yang H, Bell TA, Churchill GA, Pardo-Manuel de Villena F: On the subspecific origin of the laboratory mouse. Nat Genet 2007, 39:1100-1107.

2. Guenet JL, Simon-Chazottes D, Avner PR: The use of interspecific mouse crosses for gene localization: present status and future perspectives. Curr Top Microbiol Immunol 1988, 137:13-17.

3. Biddle FG: Segregation distortion of X-linked marker genes in interspecific crosses between Mus musculus and M. spretus. Genome 1987, 29:389-392.

4. Guenet JL: The contribution of wild derived mouse inbred strains to gene mapping methodology. Curr Top Microbiol Immunol 1986, 127:109-113.

5. Guenet $J$, Bonhomme F: Wild mice: an ever-increasing contribution to a popular mammalian model. Trends Genet 2003, 19:24-31.

6. Montagutelli X, Turner R, Nadeau JH: Epistatic control of non-Mendelian inheritance in mouse interspecific crosses. Genetics 1996, 143:1739-1752.

7. Rowe LB, Nadeau JH, Turner R, Frankel WN, Letts VA, Eppig JT, Ko MS, Thurston SJ, Birkenmeier EH: Maps from two interspecific backcross DNA panels available as a community genetic mapping resource. Mamm Genome 1994, 5:253-274.

8. Siracusa LD, Alvord WG, Bickmore WA, Jenkins NA, Copeland NG: Interspecific backcross mice show sex-specific differences in allelic inheritance. Genetics 1991, 128:813-821.

9. Ceci JD, Siracusa LD, Jenkins NA, Copeland NG: A molecular genetic linkage map of mouse chromosome 4 including the localization of several proto-oncogenes. Genomics 1989, 5:699-709. 
10. Justice MJ, Siracusa LD, Gilbert DJ, Heisterkamp N, Groffen J, Chada K, Silan CM, Copeland NG, Jenkins NA: A genetic linkage map of mouse chromosome 10: localization of eighteen molecular markers using a single interspecific backcross. Genetics 1990, 125:855-866.

11. Siracusa LD, Buchberg AM, Copeland NG, Jenkins NA: Recombinant inbred strain and interspecific backcross analysis of molecular markers flanking the murine agouti coat color locus. Genetics 1989, 122:669-679.

12. Shendure J, Melo JA, Pociask K, Derr R, Silver LM: Sex-restricted nonMendelian inheritance of mouse chromosome 11 in the offspring of crosses between $\mathrm{C} 57 \mathrm{BL} / 6 \mathrm{~J}$ and (C57BL/6J $\times$ DBA/2J)F1 mice. Mamm Genome 1998, 9:812-815.

13. Agulnik SI, Sabantsev ID, Ruvinsky AO: Effect of sperm genotype on chromatid segregation in female mice heterozygous for aberrant chromosome 1. Genet Research 1993, 61:97-100.

14. Bell TA, de la Casa-Esperón E, Doherty HE, Ideraabdullah F, Kim K, Wang Y, Lange LA, Wilhemsen K, Lange EM, Sapienza C, de Villena FP: The paternal gene of the DDK syndrome maps to the Schlafen gene cluster on mouse chromosome 11. Genetics 2006, 172:411-423.

15. Pardo-Manual de Villena F, Slamka C, Fonseca M, Naumova AK, Paquette J, Pannunzio P, Smith M, Verner A, Morgan K, Sapienza C: Transmission-ratio distortion through F1 females at chromosome 11 loci linked to Om in the mouse DDK syndrome. Genetics 1996, 142:1299-1304.

16. Pardo-Manuel de Villena F, Sapienza C: Female meiosis drives karyotypic evolution in mammals. Genetics 2001, 159:1179-1189.

17. Chevin LM, Hospital F: The hitchhiking effect of an autosomal meiotic drive gene. Genetics 2006, 173:1829-1832.

18. Malik HS: Mimulus finds centromeres in the driver's seat. Trends Ecol Evol 2005, 20:151-154.

19. Pardo-Manuel de Villena F, de la Casa-Esperon E, Briscoe TL, Sapienza C: A genetic test to determine the origin of maternal transmission ratio distortion. Meiotic drive at the mouse Om locus. Genetics 2000, 154:333-342.

20. Babinet C, Richoux V, Guenet $J$, Renard JP: The DDK inbred strain as a model for the study of interactions between parental genomes and egg cytoplasm in mouse preimplantation development. Development 1990, 81-87.

21. Sandler L, Hiraizumi Y: Meiotic Drive In Natural Populations Of Drosophila melanogaster. II. Genetic Variation At The Segregation-Distorter Locus. Proc Natl Acad Sci USA 1959, 45:1412-1422.

22. Lyon MF: Elucidating mouse transmission ratio distortion. Nat Genet 2005, 37:924-925.

23. Lyon MF, Meredith R: Investigations Of The Nature Of T-Alleles In The Mouse. I. Genetic Analysis Of A Series Of Mutants Derived From A Lethal Allele. Heredity 1964, 19:301-312.

24. Lyon MF, Meredith R: Investigations Of The Nature Of T-Alleles In The Mouse. II. Genetic Analysis Of An Unusual Mutant Allele And Its Derivatives. Heredity 1964, 19:313-325.

25. Silver LM: The Peculiar Journey Of A Selfish Chromosome: Mouse t Haplotypes And Meiotic Drive. Trends Genet 1993, 9:250-254.

26. Caspary T, Cleary MA, Baker CC, Guan XJ, Tilghman SM: Multiple mechanisms regulate imprinting of the mouse distal chromosome 7 gene cluster. Mol Cell Biol 1998, 18:3466-3474.

27. Horsthemke $B$, Wagstaff J: Mechanisms of imprinting of the Prader-Willi/ Angelman region. Am J Med Genet 2008, 146A:2041-2052.

28. Gaultier C, Gallego J: Neural control of breathing: insights from genetic mouse models. J Appl Physiol 2008, 104:1522-1530.

29. Coan PM, Burton GJ, Ferguson-Smith AC: Imprinted genes in the placenta-a review. Placenta 2005, 26:S10-S20.

30. Wu MY, Chen KS, Bressler J, Hou A, Tsai TF, Beaudet AL: Mouse imprinting defect mutations that model Angelman syndrome. Genesis 2006, 44:12-22.

31. Vrana PB, Matteson PG, Schmidt JV, Ingram RS, Joyce A, Prince $K L$, Dewey MJ, Tilghman SM: Genomic imprinting of a placental lactogen gene in Peromyscus. Dev Genes Evol 2001, 211:523-532.

32. Duselis AR, Wiley CD, O'Neill MJ, Vrana PB: Genetic evidence for a maternal effect locus controlling genomic imprinting and growth. Genesis 2005, 43:155-165.

33. Vrana PB, Guan XJ, Ingram RS, Tilghman SM: Genomic imprinting is disrupted in interspecific Peromyscus hybrids. Nat Genet 1998, 20:362-365.
34. Vrana PB, Fossella JA, Matteson P, del Rio T, O'Neill MJ, Tilghman SM: Genetic and epigenetic incompatibilities underlie hybrid dysgenesis in Peromyscus. Nat Genet 2000, 25:120-124.

35. Roberts A, Pardo-Manuel de Villena F, Wang W, McMillan L, Threadgill DW: The polymorphism architecture of mouse genetic resources elucidated using genome-wide resequencing data: implications for QTL discovery and systems genetics. Mamm Genome 2007, 18:473-481.

36. van Boven $M$, Weissing FJ: Competition at the mouse t complex: rare alleles are inherently favored. Theor Popul Biol 2001, 60:343-358.

37. de la Casa-Esperon E, Pardo-Manuel de Villena F, Verner AE, Briscoe TL, Malette JM, Rosa M, Jin WH, Sapienza C: Sex-of-offspring-specific transmission ratio distortion on mouse chromosome X. Genetics 2000, 154:343-350.

38. Innis MA, Gelfand DH, Sninsky JJ, White TJ: PCR Protocols: A Guide to Methods and Applications. San Diego: Academic Press; 1990.

39. Maniatis T, Fritsch EF, Sambroock JJ: Molecular Cloning: A Laboratory Manual. Cold Spring Harbor: Cold Spring Harbor Laboratory Press; 1982.

40. Lander E, Kruglyak L: Genetic dissection of complex traits: guidelines for interpreting and reporting linkage results. Nat Genet 1995, 11:241-247.

doi:10.1186/1471-2156-11-98

Cite this article as: Eversley et al: Genetic mapping and developmental timing of transmission ratio distortion in a mouse interspecific backcross. BMC Genetics 2010 11:98.

\section{Submit your next manuscript to BioMed Central and take full advantage of:}

- Convenient online submission

- Thorough peer review

- No space constraints or color figure charges

- Immediate publication on acceptance

- Inclusion in PubMed, CAS, Scopus and Google Scholar

- Research which is freely available for redistribution

Submit your manuscript at www.biomedcentral.com/submit
C) Biomed Central 